
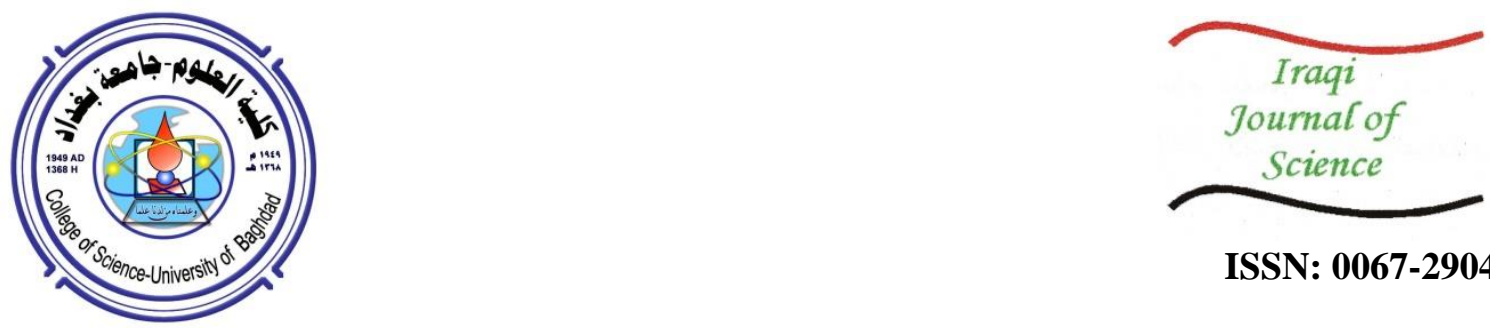

ISSN: 0067-2904

\title{
Some Properties of Fuzzy Inner Product Space
}

\author{
Jehad R. Kider
}

Branch of Mathematics and Computer Applications, Department of Applied Sciences, University of Technology

$$
\text { Received: 3/11/2020 }
$$

Accepted: 13/1/2021

\begin{abstract}
Our goal in the present paper is to introduce a new type of fuzzy inner product space. After that, to illustrate this notion, some examples are introduced. Then we prove that that every fuzzy inner product space is a fuzzy normed space. We also prove that the cross product of two fuzzy inner spaces is again a fuzzy inner product space. Next, we prove that the fuzzy inner product is a non decreasing function. Finally, if $\mathrm{U}$ is a fuzzy complete fuzzy inner product space and D is a fuzzy closed subspace of $U$, then we prove that $U$ can be written as a direct sum of $D$ and the fuzzy orthogonal complement $D^{\perp^{F}}$ of D.
\end{abstract}

Keywords: Fuzzy inner product space, Fuzzy orthogonal vectors, Fuzzy orthogonal Complement.

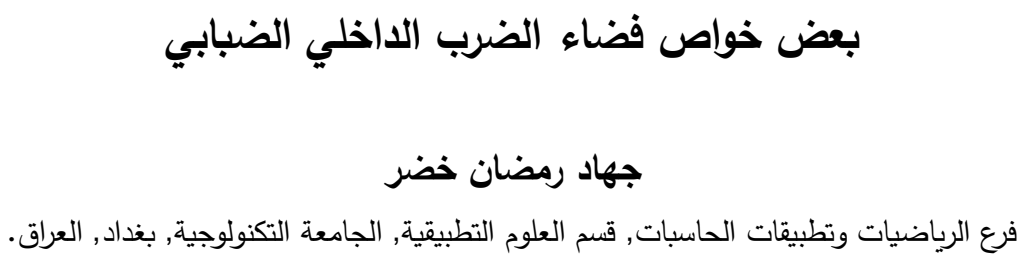

الخلاصة

هدفنا في هذا البحث هو تقديم نوع جديد من فضاء الضرب الداخلي الضبابي بعد ذلك لتوضيح هذا المفهوم

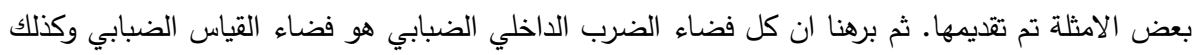

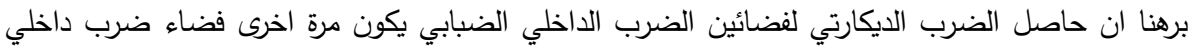

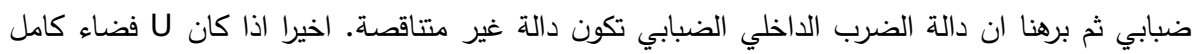

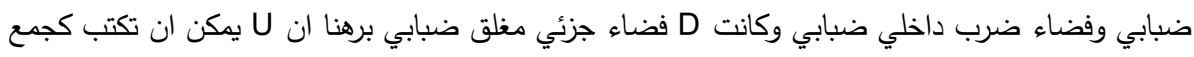

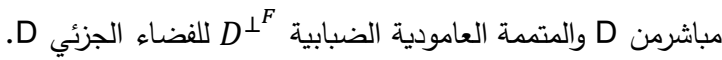

\section{Introduction}

It is known that probabilistic inner product spaces is a generalization of inner product spaces. This type of spaces are defined in [1]. Also, in a special case, the notion of a probabilistic Hilbert spaces was introduced in [2]. Other researcher introduced the fuzzy inner product spaces [3-6].

The fuzzy topological structure of a fuzzy normed space was studied by Sadeqi and Kia in 2009 [7]. Kider introduced a fuzzy normed space in 2011 [8]. Also he proved that this fuzzy normed space has a completion [9]. Again, Kider introduced a new fuzzy normed space in 2012 [10].

Kider and Kadhum, in 2017 [11], introduced the fuzzy norm for a fuzzy bounded operator on a fuzzy normed space and proved its basic properties. Then, other properties were proved by Kadhum in 2017 [12]. Ali, in 2018 [13], proved basic properties of complete fuzzy normed algebra. Kider and Ali, in 
2018 [14], introduced the notion of fuzzy absolute value and studied properties of finite dimensional fuzzy normed space.

The concept of general fuzzy normed space was presented by Kider and Gheeab in 2019 [15]. They proved basic properties of this space and the general fuzzy normed space GFB(V, U) [16]. Kider and Kadhum, in 2019 [17], introduced the notion of fuzzy compact linear operator and proved its basic properties.

There is a need to understand how to define fuzzy inner product space to be reasonable to get the similar results to the classical case. Therefore, we give a modification for the definition of fuzzy inner product space. After that, two examples are solved to show the existence of such type of inner space. Then, we prove some properties of this space.

\section{Preliminaries}

Definition 2.1 [8]. Suppose that $U$ is any set, a fuzzy set $\widetilde{A}$ in $U$ is equipped with a membership function, $\mu_{\widetilde{A}}: U \rightarrow[0,1]$. Then $\widetilde{A}$ is represented by $\widetilde{A}=\left\{\left(\mathrm{u}, \mu_{\widetilde{A}}(\mathrm{u})\right): \mathrm{u} \in \mathrm{U}, 0 \leq \mu_{\widetilde{\mathrm{A}}}(\mathrm{u}) \leq 1\right\}$.

Definition 2.2 [2]. Suppose that $\otimes: I \times I \rightarrow I$ is a binary operation where $I=[0,1]$, then $\otimes$ is known as a continuous triangular norm (or continuous t-norm) if for all $\alpha, \beta, \gamma, \delta \in \mathrm{I}$, the following properties are satisfied:

(1) $\alpha \otimes \beta=\beta \otimes \alpha,(2) \alpha * 1=\alpha,(3)(\alpha \otimes \beta) \otimes \gamma=\alpha \otimes(\beta \otimes \gamma)$.

(4) If $\alpha \leq \beta$ and $\gamma \leq \delta$, then $\alpha \otimes \gamma \leq \beta \otimes \delta$.

Remark 2.3 [11]. (1) If $\alpha, \beta \in(0,1)$ with $\alpha>\beta$, then we can find $\gamma \in(0,1)$ with $\alpha \otimes \gamma \geq \beta$. (2) If $\sigma$ $\in(0,1)$, then we can find $\delta \in(0,1)$ with $\delta \otimes \delta \geq \sigma$.

Definition 2.4 [12]. Let $\mathrm{V}$ be $\mathbb{F}$-space, $\otimes$ is a continuous t-norm, and $\mathrm{N}: \mathrm{V} \times[0 . \infty) \rightarrow \mathrm{I}$ is a fuzzy set. If the following conditions are satisfied for all $\mathrm{a}, \mathrm{b} \in \mathrm{V}$, and $\alpha, \beta>0$ :

(1) $\mathrm{N}(\mathrm{a}, \alpha) \geq 0,(2) \mathrm{N}(\mathrm{a}, \alpha)=1$ if and only if $\mathrm{a}=0$.

(3) $\mathrm{N}(\mathrm{ca}, \alpha)=\mathrm{N}\left(\mathrm{a}, \frac{\alpha}{|c|}\right)$ for all $\mathrm{c} \neq 0 \in \mathbb{F},(4) \mathrm{N}(\mathrm{a}, \alpha) \otimes \mathrm{N}(\mathrm{b}, \beta) \leq \mathrm{N}(\mathrm{a}+\mathrm{b}, \alpha+\beta)$.

(5) $\mathrm{N}(\mathrm{a},):.[0, \infty) \rightarrow \mathrm{I}$ is a continuous function of $\alpha$.

(6) $\lim _{\alpha \rightarrow \infty} N(a, \alpha)=1$,

then triple $(\mathrm{V}, \mathrm{N}, \otimes)$ is known as a fuzzy normed space.

Remark 2.5 [12]. Assume that $(\mathrm{V}, \mathrm{N}, \otimes)$ is a fuzzy normed space and suppose that $\in \mathrm{V}$. $\mathrm{t}>0$ and $\mathrm{q} \in(0,1)$. If $\mathrm{N}(\mathrm{a}, \mathrm{t})>(1-\mathrm{q})$, then there is $\mathrm{s}$ with $0<\mathrm{s}<\mathrm{t}$ such that $\mathrm{N}(\mathrm{a}, \mathrm{s})>(1-\mathrm{q})$.

Theorem 2.6 [18]. If $\otimes$ is a continuous t-norm, then $(1) 1 \otimes 1=1,(2) 0 \otimes 1=0=1 \otimes 0$

(3) $0 \otimes 0=0,(4) \mathrm{a} \otimes \mathrm{a} \leq \mathrm{a}$ for all $\mathrm{a} \in \mathrm{I}$.

Definition 2.7 [12]. Assume that $(V, N, \otimes)$ is a fuzzy normed space. $\operatorname{Put} F B(a, p, t)=\{b \in V: N(a-b$, $\mathrm{t})>(1-\mathrm{p})\}, \mathrm{FB}[\mathrm{a}, \mathrm{p}, \mathrm{t}]=\{\mathrm{b} \in \mathrm{V}: \mathrm{N}(\mathrm{a}-\mathrm{b}, \mathrm{t}) \geq(1-\mathrm{p})\}$. Then $\mathrm{FB}(\mathrm{a}, \mathrm{p}, \mathrm{t})$ and $\mathrm{FB}[\mathrm{a}, \mathrm{p}, \mathrm{t}]$ are known as open and closed fuzzy balls, respectively, where $a \in V$ is the center and $p \in(0,1)$ the radius.

Definition 2.8 [12]. Assume that $(\mathrm{V}, \mathrm{N}, \otimes)$ is a fuzzy normed space and $\mathrm{W} \subseteq \mathrm{V}$ is known as fuzzy bounded if there is $\mathrm{t}>0$ and $\mathrm{q} \in(0,1)$ with $\mathrm{N}(\mathrm{w}, \mathrm{t})>(1-\mathrm{q})$ for each $\mathrm{w} \in \mathrm{W}$.

Definition 2.9 [12]. A sequence $\left(v_{n}\right)$ in $(V, N, \otimes)$ is said to be converges to $v \in V$ if for each $t>$ 0 and $\mathrm{q} \in(0,1)$ we can find $\mathrm{K} \in \mathbb{N}$ with $\mathrm{N}\left(\mathrm{v}_{\mathrm{n}}-\mathrm{v}, \mathrm{t}\right)>(1-\mathrm{q})$ for all $\mathrm{n} \geq \mathrm{K}$. Or, in other words, $\lim _{n \rightarrow \infty} N\left(v_{n}-v, t\right)=1$ or $\lim _{n \rightarrow \infty} v_{n}=v$, or simply represented by $v_{n} \rightarrow v$, v is known as the limit of $\left(v_{n}\right)$.

Definition 2.10 [12]. A sequence $\left(v_{n}\right)$ in $(V, N, \otimes)$ is known as a Cauchy sequence if for all $q \in(0,1)$ $\mathrm{t}>0$ there is $\mathrm{K} \in \mathbb{N}$ with $\mathrm{N}\left(\mathrm{v}_{\mathrm{n}}-\mathrm{v}_{\mathrm{m}}, \mathrm{t}\right)>(1-\mathrm{q})$ for all $\mathrm{m}, \mathrm{n} \geq \mathrm{K}$.

Definition 2.11 [12]. Suppose that $(\mathrm{V}, \mathrm{N}, \otimes)$ is a fuzzy normed space and $\mathrm{W} \subseteq \mathrm{V}$. Then the closure of $\mathrm{W}$ is written by $\overline{\mathrm{W}}$ or $\mathrm{CL}(\mathrm{W})$, which is $\overline{\mathrm{W}}=\bigcap\{\mathrm{W} \subseteq \mathrm{B}: \mathrm{B}$ is closed in $\mathrm{V}\}$.

Lemma 2.12 [14]. Assume that $(\mathrm{V}, \mathrm{N}, \otimes)$ is a fuzzy normed space and $\mathrm{W} \subseteq \mathrm{V}$. Then $\mathrm{w} \in \overline{\mathrm{W}}$ if and only if we can find $\left(\mathrm{w}_{\mathrm{n}}\right)$ in $\mathrm{W}$ with $\mathrm{w}_{\mathrm{n}} \rightarrow \mathrm{w}$.

Definition 2.13 [14]. A fuzzy normed space $(\mathrm{V}, \mathrm{N}, \otimes)$ is known as complete if whenever $\left(\mathrm{v}_{\mathrm{n}}\right)$ is Cauchy se in $\mathrm{V}$ then we can find $\mathrm{v} \in \mathrm{V}$ with $\mathrm{v}_{\mathrm{n}} \rightarrow \mathrm{v}$.

\section{Fuzzy Inner Product Space}

Definition 3.1

Let $U$ be a vector space over the field $K$ (where $K=\mathbb{R}$ or $K=\mathbb{C}$ ) and let $\otimes$ be a continuous t-norm. Assume that $A: U \times U \times K \rightarrow I$ where $I=[0,1]$ is a fuzzy set, then $A$ is known as a fuzzy inner product if it satisfies the following conditions: 
(A1) $\mathrm{A}(\mathrm{p}+\mathrm{q}, \mathrm{w},|\alpha|+|\beta|) \geq \mathrm{A}(\mathrm{p}, \mathrm{w},|\alpha|) \otimes \mathrm{A}(\mathrm{q}, \mathrm{w},|\beta|)$,

(A2) $\mathrm{A}(\mathrm{p}, \mathrm{q},|\alpha \beta|) \geq \mathrm{A}\left(\mathrm{p}, \mathrm{p},|\alpha|^{2}\right) \otimes \mathrm{A}\left(\mathrm{q}, \mathrm{q},|\beta|^{2}\right)$,

(A3) $\mathrm{A}(\mathrm{p}, \mathrm{q}, \alpha)=\mathrm{A}(\mathrm{q}, \mathrm{p}, \bar{\alpha})$,

(A4) $\mathrm{A}(\mathrm{sp}, \mathrm{q}, \alpha)=\mathrm{A}\left(\mathrm{p}, \mathrm{q}, \frac{\alpha}{|s|}\right)$ for all $s \neq 0 \in \mathrm{K}$,

(A5) $\mathrm{A}(\mathrm{p}, \mathrm{p}, \alpha)=0$ for all $\alpha \in \mathbb{C}-\mathbb{R}$,

(A6) For all $\alpha \in[0, \infty), \mathrm{A}(\mathrm{p}, \mathrm{p}, \alpha)=1$ if and only if $\mathrm{p}=0$,

(A7) $\mathrm{A}(\mathrm{p}, \mathrm{q}):, \mathbb{R} \rightarrow \mathrm{I}$ is a continuous function,

(A8) $\lim _{\alpha \rightarrow \infty} A(u, v, \alpha)=1$,

for all $\mathrm{p}, \mathrm{q}, \mathrm{w} \in \mathrm{U}$. Then $(\mathrm{U}, \mathrm{A}, \otimes)$ is called a fuzzy inner product space.

Example 3.2

Let $(\mathrm{U},<., .>)$ be inner product and $\mathrm{a} \otimes \mathrm{b}=\mathrm{a} \wedge \mathrm{b}$ for all $\mathrm{a}, \mathrm{b} \in \mathrm{I}$. Define

$\mathrm{A}(\mathrm{p}, \mathrm{q}, \alpha)=\left\{\begin{array}{c}\frac{|\alpha|}{|\alpha|+|<p, q>|} \quad \text { if } \alpha \in \mathbb{C}-\{0\} \text { and } \\ u, v \text { are linearly } \\ \text { independent } \\ 0 \quad \text { if } \alpha=0 \text { and } u, v \text { are linealy } \\ \text { independent } \\ 0 \quad \text { if } \alpha \in \mathbb{C}-(0, \infty) \text { and } u, v \text { are } \\ \text { linealy independent }\end{array}\right.$

Then $(\mathrm{U}, \mathrm{A}, \otimes)$ is a fuzzy inner product space.

\section{Proof}

We will show that all conditions of definition 3.1 are satisfied.

(A1)(1) If $|\alpha|=0$ and $|\beta| \neq 0$, then $\mathrm{A}(\mathrm{p}, \mathrm{w},|\alpha|)=0$, and notice that

$|<p+q . w\rangle|=|<p, w\rangle+<q, w\rangle \mid$

$\leq|<p, w>|+|<q, w>|$. Also

$|<p+q . w>| \leq|<q, w>|$, which implies $|\beta|+|<p+q . w>| \leq|\beta|+|<q, w>|$ or

$\frac{|\beta|}{|\beta|+|<p+q . w>|} \geq \frac{|\beta|}{|\beta|+|<q, w>|}$, that is $\mathrm{A}(\mathrm{u}+\mathrm{v}, \mathrm{w},|s|) \geq \mathrm{A}(\mathrm{v}, \mathrm{w},|s|)$

Thus, $\mathrm{A}(\mathrm{p}+\mathrm{q}, \mathrm{w},|\alpha|+|\beta|) \geq \mathrm{A}(\mathrm{p}, \mathrm{w},|\alpha|) \wedge \mathrm{A}(\mathrm{q}, \mathrm{w},|\beta|)$.

(2) Similarly, we can show that

$\mathrm{A}(\mathrm{p}+\mathrm{q}, \mathrm{w},|\alpha|+|\beta|) \geq \mathrm{A}(\mathrm{p}, \mathrm{w},|\alpha|) \wedge \mathrm{A}(\mathrm{q}, \mathrm{w},|\beta|)$ when $|\alpha| \neq 0$ and $|\beta|=0$.

(3) When $|\alpha|=0$ and $|\beta|=0$, then $\mathrm{A}(\mathrm{p}+\mathrm{q}, \mathrm{w},|\alpha|+|\beta|)=0, \mathrm{~A}(\mathrm{p}, \mathrm{w},|\alpha|)=0$ and

$\mathrm{A}(\mathrm{q}, \mathrm{w},|\beta|)=0$, thus $\mathrm{A}(\mathrm{p}+\mathrm{q}, \mathrm{w},|\alpha|+|\beta|) \geq \mathrm{A}(\mathrm{p}, \mathrm{w},|\alpha|) \wedge \mathrm{A}(\mathrm{q}, \mathrm{w},|\beta|)$.

(4) Now, if $|\alpha| \neq 0$ and $|\beta| \neq 0$, then we can assume, without losing the generality,

that $\mathrm{A}(\mathrm{p}, \mathrm{w},|\alpha|) \geq \mathrm{A}(\mathrm{q}, \mathrm{w},|\beta|)$ and $|\alpha| \leq|\beta|$, which implies that

$\frac{|\alpha|}{|\alpha|+|<p, w>|} \geq \frac{|\beta|}{|\beta|+|<q, w>|}$ or $\frac{|\alpha|+|<p, w>|}{|\alpha|} \leq \frac{|\beta|+|<q, w>|}{|\beta|}$ or

$|\beta|[|\alpha|+|<p, w>|] \leq|\alpha|[|\beta|+|<q, w>|]$ or

$|\alpha||\beta|+|\alpha||<q, w>|-|\beta||\alpha|-|\beta||<p, w>| \geq 0$, that is

$|\alpha||<q, w>|-|\beta||<p, w>| \geq 0$

Now, $\mathrm{A}(\mathrm{p}+\mathrm{q}, \mathrm{w},|\alpha|+|\beta|)-\mathrm{A}(\mathrm{q}, \mathrm{w},|\beta|)=\frac{|\alpha|+|\beta|}{|\alpha|+|\beta|+|<p+q \cdot w>|}-\frac{|\beta|}{|\beta|+|<q, w>|}=$

$\underline{(|\alpha|+|\beta|)[|\beta|+|<q, w>|]-|\beta|[|\alpha|+|\beta|+|<p+q . w>|]}$

$[|\alpha|+|\beta|+|<p+q . w>|][|\beta|+|<q, w>|$

$|\alpha||\beta|+|\alpha||<q, w>|+|\beta|^{2}+|\beta||<q, w>|-|\beta||\alpha|$

$=\frac{-|\beta|^{2}-|\beta||<p+q . w>|}{[|\alpha|+|\beta|+|<p+q . w>|][|\beta|+|<q, w>|}$

$|\alpha||\beta|+|\alpha||<q, w>|+|\beta|^{2}+|\beta||<q, w>|$

$=\frac{-|\beta||\alpha|-|\beta|^{2}-|\beta||<p . w>|-|\beta||<q . w>|}{[|\alpha|+|\beta|+|<p+q . w>|][|\beta|+|<q, w>|}$

$=\frac{|\alpha||<q, w>|-|\beta||<p \cdot w>|}{[|\alpha|+|\beta|+|<p+q \cdot w>|][|\beta|+|<q, w>|} \geq 0$

Since, from equation (1), we obtain

$|\alpha||<q, w>|-|\beta||<p, w>| \geq 0$. Thus, $\mathrm{A}(\mathrm{p}+\mathrm{q}, \mathrm{w},|\alpha|+|\beta|) \geq \mathrm{A}(\mathrm{q}, \mathrm{w},|\beta|)$

But $\mathrm{A}(\mathrm{p}, \mathrm{w},|\alpha|) \geq \mathrm{A}(\mathrm{v}, \mathrm{w},|\beta|)$ by our assumption, hence we have

$\mathrm{A}(\mathrm{p}+\mathrm{q}, \mathrm{w},|\alpha|+|\beta|) \geq \mathrm{A}(\mathrm{p}, \mathrm{w},|\alpha|) \wedge \mathrm{A}(\mathrm{q}, \mathrm{w},|\beta|)$. 
(A2) To show that $\mathrm{A}(\mathrm{u}, \mathrm{v},|\alpha \beta|) \geq \mathrm{A}\left(\mathrm{p}, \mathrm{p},|\alpha|^{2}\right) \wedge \mathrm{A}\left(\mathrm{q}, \mathrm{q},|\beta|^{2}\right)$;

(1) if $\alpha=0$ or $\beta=0$, then $\alpha \beta=0$, which implies that $\mathrm{A}(\mathrm{p}, \mathrm{q},|\alpha \beta|)=1, \mathrm{~A}\left(\mathrm{p}, \mathrm{p},|\alpha|^{2}\right)=1$

or $\mathrm{A}\left(\mathrm{q}, \mathrm{q},|\beta|^{2}\right)=1$. Hence, $\mathrm{A}(\mathrm{p}, \mathrm{q},|\alpha \beta|) \geq \mathrm{A}\left(\mathrm{p}, \mathrm{p},|\alpha|^{2}\right) \wedge \mathrm{A}\left(\mathrm{q}, \mathrm{q},|\beta|^{2}\right)$.

(2) If $\alpha=0$ and $\alpha=0$, then $\alpha \beta=0$, which implies that $\mathrm{A}(\mathrm{p}, \mathrm{q},|\alpha \beta|)=1, \mathrm{~A}\left(\mathrm{p}, \mathrm{p},|\alpha|^{2}\right)=1$

and $\mathrm{A}\left(\mathrm{q}, \mathrm{q},|\beta|^{2}\right)=1$. Hence, $\mathrm{A}(\mathrm{p}, \mathrm{q},|\alpha \beta|) \geq \mathrm{A}\left(\mathrm{p}, \mathrm{p},|\alpha|^{2}\right) \wedge \mathrm{A}\left(\mathrm{q}, \mathrm{q},|\beta|^{2}\right)$.

(3) If $\alpha \neq 0$ and $\beta \neq 0$, then $|\alpha| \neq 0,|\beta| \neq 0$ so $|\alpha \beta| \neq 0$. Without loss of generality, assume

that $\mathrm{A}\left(\mathrm{p}, \mathrm{p},|\alpha|^{2}\right) \geq \mathrm{A}\left(\mathrm{q}, \mathrm{q},|\beta|^{2}\right)$ so $\frac{|\alpha|^{2}}{|\alpha|^{2}+|<p, p>|} \geq \frac{|\beta|^{2}}{|\beta|^{2}+|<q, q>|}$ or $\frac{|\alpha|^{2}+|<p, p>|}{|\alpha|^{2}} \leq \frac{|\beta|^{2}+|<q, q>|}{|\beta|^{2}}$

that is $\frac{|<p, p>|}{|\alpha|^{2}} \leq \frac{|<q, q>|}{|\beta|^{2}}$ or $\frac{|<p, p>||<q, q>|}{|\alpha|^{2}} \leq \frac{|<q, q>|^{2}}{|\beta|^{2}}$ that is $\frac{|<p, p>||<q, q>|}{|\beta|^{2}|\alpha|^{2}} \leq \frac{|<q, q>|^{2}}{|\beta|^{4}}$ or

$\frac{|<p, q>|^{2}}{|\beta|^{2}|\alpha|^{2}} \leq \frac{|<q, q>|^{2}}{|\beta|^{4}}$, which implies that $\frac{|<p, q>|}{|\beta||\alpha|} \leq \frac{|<q, q>|}{|\beta|^{2}}$ or $\frac{|t s|+|<p, q>|}{|\alpha \beta|} \leq \frac{|s|^{2}+|<q, q>|}{|\beta|^{2}}$, thus $\frac{|\alpha \beta|}{|\alpha \beta|+|<p, q>|} \geq$ $\frac{|\beta|^{2}}{|\beta|^{2}+|<q, q>|}$. Hence, $\mathrm{A}(\mathrm{p}, \mathrm{q},|\alpha \beta|) \geq \mathrm{A}\left(\mathrm{q}, \mathrm{q},|\beta|^{2}\right)$, but

$\mathrm{A}\left(\mathrm{p}, \mathrm{p},|\alpha|^{2}\right) \geq \mathrm{A}\left(\mathrm{q}, \mathrm{q},|\beta|^{2}\right)$ by our assumption, thus $\mathrm{A}(\mathrm{p}, \mathrm{q},|\alpha \beta|) \geq \mathrm{A}\left(\mathrm{p}, \mathrm{p},|\alpha|^{2}\right) \wedge \mathrm{A}\left(\mathrm{q}, \mathrm{q},|\beta|^{2}\right)$.

(A3) To show that $\mathrm{A}(\mathrm{p}, \mathrm{q}, \alpha)=\mathrm{A}(\mathrm{q}, \mathrm{p}, \bar{\alpha})$ we know that $|\alpha|=|\bar{\alpha}|$ for all $\alpha \in \mathbb{C}$. Now

$\mathrm{A}(\mathrm{p}, \mathrm{q}, \alpha)=\frac{|\alpha|}{|\alpha|+|<p, q>|}=\frac{|\bar{\alpha}|}{|\bar{\alpha}|+|<q, p>|}=\mathrm{A}(\mathrm{q}, \mathrm{p}, \bar{\alpha})$.

(A4) To show that $\mathrm{A}(\mathrm{sp}, \mathrm{q}, \alpha)=\mathrm{A}\left(\mathrm{p}, \mathrm{q}, \frac{\alpha}{|s|}\right)$ for all $\mathrm{s} \neq 0 \in \mathrm{K}$.

(1) When $\alpha \neq 0 \in \mathbb{C}$ and $p, q$ are linearly independent, here we have two cases. Case one, if $<p$, $\mathrm{q}>=0$,

then $\mathrm{A}(\mathrm{sp}, \mathrm{q}, \alpha)=\frac{|\alpha|}{|\alpha|+|<s p, q>|}=\frac{|\alpha|}{|\alpha|+|s||p, q>|}=\frac{|\alpha|}{|\alpha|}=1$. Also, $\mathrm{A}\left(\mathrm{p}, \mathrm{q}, \frac{\alpha}{|s|}\right)=\frac{\frac{\alpha}{|s|}}{\frac{\alpha}{|s|}+|<p, q>|}=\frac{\frac{\alpha}{|s|}}{\frac{\alpha}{|s|}}=1$. Thus $\mathrm{A}(\mathrm{sp}$, $\mathrm{q}, \mathrm{t})=\mathrm{A}\left(\mathrm{p}, \mathrm{q}, \frac{\alpha}{|s|}\right)$. Case two, if $\langle\mathrm{p}, \mathrm{q}>\neq 0$, then

$<\mathrm{sp}, \mathrm{q}>=\mathrm{s}<\mathrm{p}, \mathrm{q}>\neq 0$ if $\mathrm{s} \neq 0$. Now,

$\mathrm{A}(\mathrm{sp}, \mathrm{q}, \alpha)=\frac{|\alpha|}{|\alpha|+|<\alpha p, q>|}=\frac{|\alpha|}{|\alpha|+|s||<p, q>|}=\frac{\frac{\alpha}{|s|}}{\frac{\alpha}{|s|}+|<p, q>|}=\mathrm{A}\left(\mathrm{p}, \mathrm{q}, \frac{\alpha}{|s|}\right)$.

(2) When $\alpha=0$ and $\mathrm{u}, \mathrm{v}$ are linearly independent, then here we have

$\mathrm{A}(\mathrm{sp}, \mathrm{q}, \alpha)=0=\mathrm{A}\left(\mathrm{p}, \mathrm{q}, \frac{\alpha}{|s|}\right)$.

(A5) Now, $\mathrm{A}(\mathrm{p}, \mathrm{p}, \alpha)=0$ for all $\alpha \in \mathbb{C}-\mathbb{R}$ follows immediately from our definition.

(A6) We prove that when $\alpha \in[0, \infty), \mathrm{A}(\mathrm{p}, \mathrm{p}, \alpha)=1$ if and only if $\mathrm{p}=0$. Now,

$\mathrm{A}(\mathrm{p}, \mathrm{p}, \alpha)=1$ if and only if $\frac{|\alpha|}{|\alpha|+|<p, p>|}=1$, if and only if $|<p, p>|=0$, if and only if $\mathrm{p}=0$.

(A7) To show that $\mathrm{A}(\mathrm{p}, \mathrm{q}):, \mathbb{R} \rightarrow \mathrm{I}$ is a continuous function, let $\left(\alpha_{n}\right)$ be a sequence in $\mathbb{R}$ such that $\alpha_{n} \rightarrow \alpha \in \mathbb{R}$. Now, $\lim _{n \rightarrow \infty} A\left(p, q, \alpha_{n}\right)=\lim _{n \rightarrow \infty} \frac{\left|\alpha_{n}\right|}{\left|\alpha_{n}\right|+|<p, q>|}=\frac{|\alpha|}{|\alpha|+|<p, q>|}=\mathrm{A}(\mathrm{p}, \mathrm{q}, \alpha)$. This means that $\lim _{n \rightarrow \infty} \mathrm{A}\left(\mathrm{p}, \mathrm{q}, \alpha_{n}\right)=\mathrm{A}(\mathrm{p}, \mathrm{q}, \alpha)$. Hence, $\mathrm{A}(\mathrm{p}, \mathrm{q}):, \mathbb{R} \rightarrow \mathrm{I}$ is a

continuous function.

(A8) Finally, we will show that $\lim _{\alpha \rightarrow \infty} A(p, q, \alpha)=1$. Now,

$\lim _{\alpha \rightarrow \infty} A(p, q, \alpha)=\lim _{\alpha \rightarrow \infty} \frac{|\alpha|}{|\alpha|+|<p, q>|}=\lim _{\alpha \rightarrow \infty} \frac{1}{1+\frac{|<p, q>|}{|\alpha|}}=1$.

Hence, $(\mathrm{U}, \mathrm{A}, \otimes)$ is a fuzzy inner product space.

Example 3.3

Let $(\mathrm{U},<., .>)$ be inner product space and $\mathrm{a} \otimes \mathrm{b}=\mathrm{a} \wedge \mathrm{b}$ for all $\mathrm{a}, \mathrm{b} \in \mathrm{I}$. Define

$\mathrm{A}(\mathrm{p}, \mathrm{q}, \alpha)=\left\{\begin{array}{rr}1 & \text { if } \alpha \leq 1<p, q>1 \\ 0 & \text { if } \alpha>\mid<p, q>1 \\ 0 & \text { if } \alpha \in \mathbb{C}-\mathbb{R}\end{array}\right.$

Then, $(U, A, \otimes)$ is a fuzzy inner product space.

Proof

We will show that all conditions of definition 3.1 are satisfied.

(A1) First, we will show that $\mathrm{A}(\mathrm{p}+\mathrm{q}, \mathrm{w},|\alpha|+|\beta|) \geq \mathrm{A}(\mathrm{p}, \mathrm{w},|\alpha|) \wedge \mathrm{A}(\mathrm{q}, \mathrm{w},|\beta|)$.

(1) If $\alpha \leq|<p, w>|$ and $\beta \leq|<q, w>|$, then $|\alpha| \leq|<p, w>|$ and $|\beta| \leq|<q, w\rangle \mid$.

Thus, $[|\alpha|+|\beta|] \leq[|<p, w>|+|<q, w>|]=|<p+q, w>|$.

So, $\mathrm{A}(\mathrm{p}+\mathrm{q}, \mathrm{w},|\alpha|+|\beta|)=1, \mathrm{~A}(\mathrm{p}, \mathrm{w},|\alpha|)=1$, and $\mathrm{A}(\mathrm{q}, \mathrm{w},|\beta|)=1$, that is 
$\mathrm{A}(\mathrm{p}+\mathrm{q}, \mathrm{w},|\alpha|+|\beta|) \geq \mathrm{A}(\mathrm{p}, \mathrm{w},|\alpha|) \wedge \mathrm{A}(\mathrm{q}, \mathrm{w},|\beta|)$ holds.

(2) If $\alpha>|<p, w>|$ and $\beta>|<q, w>|$, then $|\alpha|>|<p, w>|$ and $|\beta|>|<q, w\rangle \mid$.

Thus, $[|\alpha|+|\beta|]\rangle[|<p, w\rangle|+|<q, w\rangle \mid]=|<p+q, w\rangle \mid$. So,

$\mathrm{A}(\mathrm{p}+\mathrm{q}, \mathrm{w},|\alpha|+|\beta|)=0, \mathrm{~A}(\mathrm{p}, \mathrm{w},|\alpha|)=0$ and $\mathrm{A}(\mathrm{q}, \mathrm{w},|\beta|)=0$, that is

$\mathrm{A}(\mathrm{p}+\mathrm{q}, \mathrm{w},|\alpha|+|\beta|) \geq \mathrm{A}(\mathrm{p}, \mathrm{w},|\alpha|) \wedge \mathrm{A}(\mathrm{q}, \mathrm{w},|\beta|)$ holds.

(3) If $\alpha \leq|<p, w\rangle \mid$ and $\alpha>|\langle q, w\rangle|$, then $|\alpha| \leq|<p, w\rangle \mid$ and $|\beta|>|<q, w\rangle \mid$.

This implies that $\mathrm{A}(\mathrm{p}, \mathrm{w},|\alpha|)=1, \mathrm{~A}(\mathrm{q}, \mathrm{w},|\beta|)=0$, so $\mathrm{A}(\mathrm{p}, \mathrm{w},|\alpha|) \wedge \mathrm{A}(\mathrm{q}, \mathrm{w},|\beta|)=1 \wedge 0=0$.

But $\mathrm{A}(\mathrm{p}+\mathrm{q}, \mathrm{w},|\alpha|+|\beta|) \geq 0$. Thus $\mathrm{A}(\mathrm{p}+\mathrm{q}, \mathrm{w},|\alpha|+|\beta|) \geq \mathrm{A}(\mathrm{p}, \mathrm{w},|\alpha|) \wedge \mathrm{A}(\mathrm{q}, \mathrm{w},|\beta|)$ holds.

(4) The case $\alpha>|<p, w\rangle \mid$ and $\beta \leq|<q, w\rangle \mid$ is similar to the case (3) and hence is omitted.

(A2) To show that $\mathrm{A}(\mathrm{p}, \mathrm{q},|\alpha \beta|) \geq \mathrm{A}\left(\mathrm{q}, \mathrm{p},|\alpha|^{2}\right) \wedge \mathrm{A}\left(\mathrm{q}, \mathrm{q},|\beta|^{2}\right)$.

(1) If $|\alpha|^{2} \leq|<p, p>|$ and $|\beta|^{2} \leq|<q, q>|$, then

$|\alpha|^{2}|\beta|^{2} \leq|<p, p>||<q, q>|=\|p\|^{2}\|q\|^{2}=|<p, q>|^{2}$ or

$|\alpha \beta|^{2} \leq|<p, q>|^{2}$, which implies that $|\alpha \beta| \leq|<p, q>|$. Thus, $\mathrm{A}(\mathrm{p}, \mathrm{q},|\alpha \beta|)=1, \mathrm{~A}\left(\mathrm{p}, \mathrm{p},|\alpha|^{2}\right)=1$ and $\mathrm{A}\left(\mathrm{q}, \mathrm{q},|\beta|^{2}\right)=1$, that is $\mathrm{A}(\mathrm{p}, \mathrm{q},|\alpha \beta|) \geq \mathrm{A}\left(\mathrm{p}, \mathrm{p},|\alpha|^{2}\right) \wedge \mathrm{A}\left(\mathrm{q}, \mathrm{q},|\beta|^{2}\right)$ holds.

(2) If $|\alpha|^{2}>|<p, p>|$ and $|\beta|^{2}>|<q, q>|$, then $|\alpha|^{2}|\alpha|^{2}>|<p, p>||<q, q>|=\|p\|^{2}\|q\|^{2}=|<p, q>|^{2}$, or

$|\alpha \beta|^{2}>|<p, q>|^{2}$, which implies that $|\alpha \beta|>|<p, q>|$. Thus, $\mathrm{A}(\mathrm{p}, \mathrm{q},|\alpha \beta|)=0, \mathrm{~A}\left(\mathrm{p}, \mathrm{p},|\alpha|^{2}\right)=0$ and $\mathrm{A}\left(\mathrm{q}, \mathrm{q},|\beta|^{2}\right)=0$, that is $\mathrm{A}(\mathrm{p}, \mathrm{q},|\alpha \beta|) \geq \mathrm{A}\left(\mathrm{p}, \mathrm{p},|\alpha|^{2}\right) \wedge \mathrm{A}\left(\mathrm{q}, \mathrm{q},|\beta|^{2}\right)$ holds.

(3) If $|\alpha|^{2} \leq|<p, p>|$ and $|\beta|^{2}>|<q, q>|$, then $\mathrm{A}\left(\mathrm{p}, \mathrm{p},|\alpha|^{2}\right)=1$ and $\mathrm{A}\left(\mathrm{q}, \mathrm{q},|\beta|^{2}\right)=0$, so $\mathrm{A}\left(\mathrm{p}, \mathrm{p},|\alpha|^{2}\right) \wedge \mathrm{A}\left(\mathrm{q}, \mathrm{q},|\beta|^{2}\right)=1 \wedge 0=0$. But $\mathrm{A}(\mathrm{p}, \mathrm{q},|\alpha \beta|) \geq 0$, that is $\mathrm{A}(\mathrm{p}, \mathrm{q},|\alpha \beta|) \geq \mathrm{A}\left(\mathrm{p}, \mathrm{p},|\alpha|^{2}\right) \wedge \mathrm{A}\left(\mathrm{q}, \mathrm{q},|\beta|^{2}\right)$.

(4) The case $|\alpha|^{2}>|<p, p>|$ and $|\beta|^{2} \leq|<q, q>|$ is similar to case (3) and hence is omitted.

(A3) $\mathrm{A}(\mathrm{p}, \mathrm{q}, \alpha)=\mathrm{A}(\mathrm{q}, \mathrm{p}, \bar{\alpha})$ follows immediately from our definition.

(A4) $\mathrm{A}(\mathrm{sp}, \mathrm{q}, \alpha)=\mathrm{A}\left(\mathrm{p}, \mathrm{q}, \frac{\alpha}{|s|}\right)$ for all $\mathrm{s} \neq 0 \in \mathrm{K}$ follows from the property

$|<s p, q>|=|s||<p, q>|$.

(A5) $\mathrm{A}(\mathrm{p}, \mathrm{p}, \alpha)=0$ for all $\alpha \in \mathbb{C}-\mathbb{R}$ follows immediately from our definition.

(A6) We prove that $\alpha \in[0, \infty), \mathrm{A}(\mathrm{p}, \mathrm{p}, \alpha)=1$ if and only if $\mathrm{p}=0$. Now, if $\mathrm{p}=0$, then $|<p, p\rangle \mid=0$ and $\alpha \leq|<p, p>|$, implies $\mathrm{A}(\mathrm{p}, \mathrm{p}, \mathrm{t})=1$. But if $\mathrm{A}(\mathrm{p}, \mathrm{p}, \mathrm{t})=1$,

then $|<p, p\rangle \mid \geq \alpha \geq 0$, which implies that $|<p, p\rangle \mid=0$ so $\mathrm{p}=0$.

(A7) $\mathrm{A}(\mathrm{p}, \mathrm{q}):, \mathbb{R} \rightarrow \mathrm{I}$ is a continuous function, which is clear.

(A8) It is clear that $\lim _{\alpha \rightarrow \infty} A(p, q, \alpha)=1$.

Hence $(U, A, \Lambda)$ is a fuzzy inner product space.

The next result shows that every fuzzy inner product space is a fuzzy normed space.

\section{Theorem 3.4}

If $(\mathrm{U}, \mathrm{A}, \otimes)$ is a fuzzy inner product space where $\mathrm{a} \otimes \mathrm{b}=\mathrm{a} \wedge \mathrm{b}$ for all $\mathrm{a}, \mathrm{b} \in \mathrm{I}$, when we define

$N_{A}(\mathrm{p}, \alpha)=\left\{\begin{array}{cl}A\left(p, p, \alpha^{2}\right) & \text { if } \alpha \in(0, \infty) \\ 1 & \text { if } \alpha \leq 0\end{array}\right.$,

then $\left(\mathrm{U}, N_{A}, \otimes\right)$ is a fuzzy normed space.

\section{Proof}

To check that all the conditions of definition 2.4 are satisfied.

(1) It is clear that $0 \leq N_{A}(\mathrm{p}, \alpha) \leq 1$.

(2) Also, $N_{A}(\mathrm{p}, \alpha)=1$ if and only if $\mathrm{p}=0$.

(3) Now, $N_{A}(\mathrm{sp}, \alpha)=\mathrm{A}\left(\mathrm{sp}, \mathrm{sp}, \alpha^{2}\right)=\mathrm{A}\left(\mathrm{p}, \mathrm{p}, \frac{\alpha^{2}}{|s|^{2}}\right)=N_{A}\left(\mathrm{p}, \frac{\alpha}{|s|}\right)$ for all $\mathrm{s} \neq 0 \in \mathrm{K}$.

(4) We will show that $N_{A}(\mathrm{p}+\mathrm{q}, \alpha+\beta) \geq N_{A}(\mathrm{p}, \alpha) \wedge N_{A}(\mathrm{q}, \beta)$.

We consider the following cases:

(1) When $\alpha<0$ and $\beta<0$, then $(\alpha+\beta)<0$

(2) When $(\alpha+\beta) \geq 0, \alpha \geq 0$ and $\beta<0$ or $(\alpha+\beta) \geq 0, \alpha<0$ and $\beta \geq 0$.

(3) When $(\alpha+\beta) \geq 0, \alpha \geq 0$ and $\beta \geq 0$.

For cases (1) and (2), the result is obvious. Now,

$N_{A}(\mathrm{p}+\mathrm{q}, \alpha+\beta)=\mathrm{A}\left(\mathrm{p}+\mathrm{q}, \mathrm{p}+\mathrm{q},(\alpha+\beta)^{2}\right)=\mathrm{A}\left(\mathrm{p}+\mathrm{q}, \mathrm{p}+\mathrm{q}, \alpha^{2}+\beta^{2}+\alpha \beta+\beta \alpha\right)$ 
$\geq \mathrm{A}\left(\mathrm{p}, \mathrm{p}, \alpha^{2}\right) \wedge \mathrm{A}\left(\mathrm{q}, \mathrm{q}, \beta^{2}\right) \wedge \mathrm{A}(\mathrm{p}, \mathrm{q}, \alpha \beta) \wedge \mathrm{A}(\mathrm{q}, \mathrm{p}, \beta \alpha)$

$\geq \mathrm{A}\left(\mathrm{p}, \mathrm{p}, \alpha^{2}\right) \wedge \mathrm{A}\left(\mathrm{q}, \mathrm{q}, \beta^{2}\right) \geq N_{A}(\mathrm{p}, \alpha) \wedge N_{A}(\mathrm{q}, \beta)$.

(N5) is clear.

Hence, $\left(\mathrm{U}, N_{A}, \bigotimes\right)$ is a fuzzy normed space.

Theorem 3.5

If $\left(\mathrm{U}, A_{1}, \otimes\right)$ and $\left(\mathrm{U}, A_{2}, \otimes\right)$ are two fuzzy inner product spaces, where $\alpha \otimes \beta=\min \{\alpha, \beta\}$, where all $\alpha, \beta \in \mathrm{I}$, then $(\mathrm{U}, \mathrm{A}, \otimes)$ is also a fuzzy inner product space when we define $\mathrm{A}(\mathrm{p}, \mathrm{q}, \alpha)=A_{1}(\mathrm{p}, \mathrm{q}, \alpha) \otimes$ $A_{2}(\mathrm{p}, \mathrm{q}, \alpha)$ for all $\mathrm{u}, \mathrm{v} \in \mathrm{U}$.

\section{Proof}

We will show that all conditions of definition 3.1 are satisfied.

(A1) First, we will show that $\mathrm{A}(\mathrm{p}+\mathrm{q}, \mathrm{w},|\alpha|+|\beta|) \geq \mathrm{A}(\mathrm{p}, \mathrm{w},|\alpha|) \wedge \mathrm{A}(\mathrm{q}, \mathrm{w},|\beta|)$.

$\mathrm{A}(\mathrm{p}+\mathrm{q}, \mathrm{w},|\alpha|+|\beta|)=A_{1}(\mathrm{p}+\mathrm{q}, \mathrm{w},|\alpha|+|\beta|) \wedge A_{2}(\mathrm{p}+\mathrm{q}, \mathrm{w},|\alpha|+|\beta|)$

$\geq A_{1}(\mathrm{p}, \mathrm{w},|\alpha|) \wedge A_{1}(\mathrm{q}, \mathrm{w},|\beta|) \wedge A_{2}(\mathrm{p}, \mathrm{w},|\alpha|) \wedge A_{2}(\mathrm{q}, \mathrm{w},|\beta|)$

$=\left[A_{1}(\mathrm{p}, \mathrm{w},|\alpha|) \wedge A_{2}(\mathrm{p}, \mathrm{w},|\alpha|)\right] \wedge\left[A_{1}(\mathrm{q}, \mathrm{w},|\beta|) \wedge A_{2}(\mathrm{q}, \mathrm{w},|\beta|)\right]$

$=\mathrm{A}(\mathrm{p}, \mathrm{w},|\alpha|) \wedge \mathrm{A}(\mathrm{q}, \mathrm{w},|\beta|)$.

(A2) To show that $\mathrm{A}(\mathrm{p}, \mathrm{q},|\alpha \beta|) \geq \mathrm{A}\left(\mathrm{p}, \mathrm{p},|\alpha|^{2}\right) \wedge \mathrm{A}\left(\mathrm{q}, \mathrm{q},|\beta|^{2}\right)$. Now,

$\mathrm{A}(\mathrm{p}, \mathrm{q},|\alpha \beta|)=A_{1}(\mathrm{p}, \mathrm{q},|\alpha \beta|) \wedge A_{2}(\mathrm{p}, \mathrm{q},|\alpha \beta|)$

$\geq A_{1}\left(\mathrm{p}, \mathrm{p},|\alpha|^{2}\right) \wedge A_{1}\left(\mathrm{q}, \mathrm{q},|\beta|^{2}\right) \wedge A_{2}\left(\mathrm{p}, \mathrm{p},|\alpha|^{2}\right) \wedge A_{2}\left(\mathrm{q}, \mathrm{q},|\beta|^{2}\right)$

$\geq\left[A_{1}\left(\mathrm{p}, \mathrm{p},|\alpha|^{2}\right) \wedge A_{2}\left(\mathrm{p}, \mathrm{p},|\alpha|^{2}\right)\right] \wedge\left[A_{1}\left(\mathrm{q}, \mathrm{q},|\beta|^{2}\right) \wedge A_{2}\left(\mathrm{q}, \mathrm{q},|\beta|^{2}\right)\right]$

$=\mathrm{A}\left(\mathrm{p}, \mathrm{p},|\alpha|^{2}\right) \wedge \mathrm{A}\left(\mathrm{q}, \mathrm{q},|\beta|^{2}\right)$.

(A3) To show that $\mathrm{A}(\mathrm{p}, \mathrm{q}, \alpha)=\mathrm{A}(\mathrm{q}, \mathrm{p}, \bar{\alpha})$. We know that

$\mathrm{A}(\mathrm{p}, \mathrm{q}, \alpha)=A_{1}(\mathrm{p}, \mathrm{q}, \alpha) \wedge A_{2}(\mathrm{p}, \mathrm{q}, \alpha)=A_{1}(\mathrm{q}, \mathrm{p}, \bar{\alpha}) \wedge A_{2}(\mathrm{q}, \mathrm{p}, \bar{\alpha})=\mathrm{A}(\mathrm{q}, \mathrm{p}, \bar{\alpha})$.

(A4) To show that $\mathrm{A}(\mathrm{sp}, \mathrm{q}, \alpha)=\mathrm{A}\left(\mathrm{p}, \mathrm{q}, \frac{\alpha}{|s|}\right)$ for all $\mathrm{s} \neq 0 \in \mathrm{K}$. Now, $\mathrm{A}(\mathrm{sp}, \mathrm{q}, \alpha)=A_{1}(\mathrm{sp}, \mathrm{q}, \alpha) \wedge A_{2}$ (sp, $\mathrm{q}, \alpha)=A_{1}\left(\mathrm{p}, \mathrm{q}, \frac{\alpha}{|s|}\right) \wedge A_{2}\left(\mathrm{p}, \mathrm{q}, \frac{\alpha}{|s|}\right)=\mathrm{A}\left(\mathrm{p}, \mathrm{q}, \frac{\alpha}{|s|}\right)$.

(A5) To show that $\mathrm{A}(\mathrm{p}, \mathrm{p}, \alpha)=0$ for all $\alpha \in \mathbb{C}-\mathbb{R}$. Let

$\mathrm{A}(\mathrm{p}, \mathrm{p}, \alpha)=A_{1}(\mathrm{p}, \mathrm{p}, \alpha) \wedge A_{2}(\mathrm{p}, \mathrm{p}, \alpha)=0 \wedge 0=0$ for all $\alpha \in \mathbb{C}-\mathbb{R}$.

(A6) To prove that when $\alpha \in[0, \infty), \mathrm{A}(\mathrm{p}, \mathrm{p}, \alpha)=1$ if and only if $\mathrm{p}=0$, then

$\mathrm{A}(\mathrm{p}, \mathrm{p}, \alpha)=1$ if and only if $A_{1}(\mathrm{p}, \mathrm{p}, \alpha) \wedge A_{2}(\mathrm{p}, \mathrm{p}, \alpha)=1$, if and only if $A_{1}(\mathrm{p}, \mathrm{p}, \alpha)=1$ and $A_{2}(\mathrm{p}, \mathrm{p}$, $\alpha)=1$, if and only if $\mathrm{p}=0$.

(A7) It is clear that $\mathrm{A}(\mathrm{p}, \mathrm{q}):, \mathbb{R} \rightarrow \mathrm{I}$ is a continuous function since

$A_{1}(\mathrm{p}, \mathrm{q}):, \mathbb{R} \rightarrow \mathrm{I}$ is a continuous function and $A_{2}(\mathrm{p}, \mathrm{q}):, \mathbb{R} \rightarrow \mathrm{I}$ is a continuous function.

(A8) It is clear that $\lim _{\alpha \rightarrow \infty} A(p, q, \alpha)=1$, since $\lim _{\alpha \rightarrow \infty} A_{2}(p, q, \alpha)=1$ and

$\lim _{\alpha \rightarrow \infty} A_{1}(p, q, \alpha)=1$. Hence, $(\mathrm{U}, \mathrm{A}, \Lambda)$ is a fuzzy inner product space.

The proof of the next result is similar to the proof of Theorem 3.5 and hence is omitted.

\section{Corollary 3.6}

If $\left(\mathrm{U}, A_{1}, \otimes\right),\left(\mathrm{U}, A_{2}, \otimes\right), \ldots,\left(\mathrm{U}, A_{n}, \otimes\right)$ are fuzzy inner product spaces where $\alpha \otimes \beta=\min \{\alpha, \beta\}$, where $\alpha, \beta \in \mathrm{I}$, then $(\mathrm{U}, \mathrm{A}, \otimes)$ is also a fuzzy inner product space when we define $\mathrm{A}(\mathrm{p}, \mathrm{q}, \alpha)=A_{1}(\mathrm{p}$, $\mathrm{q}, \alpha) \otimes A_{2}(\mathrm{p}, \mathrm{q}, \alpha) \otimes \ldots \otimes A_{n}(\mathrm{p}, \mathrm{q}, \alpha)$ for all $\mathrm{p}, \mathrm{q} \in \mathrm{U}$.

The proof of the next result is similar to the proof of Theorem 2.5 and hence is deleted.

\section{Theorem 3.7}

If $\left(\mathrm{U}, A_{U}, \otimes\right)$ and $\left(\mathrm{V}, A_{V}, \otimes\right)$ are two fuzzy inner product spaces where $\alpha \otimes \beta=\min \{\alpha, \beta\}$, where $\alpha$, $\beta \in \mathrm{I}$, then $(\mathrm{W}, \mathrm{A}, \otimes)$ is also a fuzzy inner product space when we define

$\mathrm{W}=\mathrm{U} \times \mathrm{V}$ and $\mathrm{A}\left[\left(p_{1}, q_{1}\right),\left(p_{2}, q_{2}\right), \alpha\right)=A_{U}\left(p_{1}, p_{2}, \alpha\right) \otimes A_{V}\left(q_{1}, q_{2}, \alpha\right)$ for all $p_{1}, p_{2} \in \mathrm{U}$ and $q_{1}, q_{2}$ $\in \mathrm{V}$.

The proof of the next result is similar to the proof of Theorem 3.7 and hence is omitted.

\section{Corollary 3.8}

If $\left(U_{1}, A_{1}, \otimes\right),\left(U_{2}, A_{2}, \otimes\right), \ldots,\left(U_{n}, A_{n}, \otimes\right)$ are fuzzy inner product spaces where $\alpha \otimes \beta=\min \{\alpha$, $\beta$, where $\alpha, \beta \in \mathrm{I}$, then $(\mathrm{U}, \mathrm{A}, \otimes)$ is also a fuzzy inner product space when we define $\mathrm{U}=U_{1} \times U_{2} \times$ $\ldots \times U_{n}$ and $\mathrm{A}\left[\left(p_{1}, p_{2}, \ldots p_{n}\right),\left(q_{1}, q_{2}, \ldots q_{n}\right), \mathrm{t}\right)=A_{1}\left(p_{1}, q, \alpha\right) \otimes$

$A_{2}\left(p_{2}, q_{2}, \alpha\right) \otimes \ldots \otimes A_{n}\left(p_{n}, v_{n}, \alpha\right)$ for all,$p_{j} \in U_{j}$ and $q_{j} \in V_{j}$ for $\mathrm{j}=1,2, \ldots, \mathrm{n}$.

The proof of the next result is straightforward and hence is deleted.

\section{Proposition 3.9}

If $(\mathrm{U}, \mathrm{A}, \otimes)$ is a fuzzy inner product space, then 
(1) $\mathrm{A}(\mathrm{w}, \mathrm{p}+\mathrm{q},|\alpha|+|\beta|) \geq \mathrm{A}(\mathrm{w}, \mathrm{p},|\alpha|) \otimes \mathrm{A}(\mathrm{w}, \mathrm{q},|\beta|)$.

(2) $\mathrm{A}(\mathrm{sp}, \mathrm{q}, \alpha)=\mathrm{A}(\mathrm{p}, \mathrm{sq}, \alpha)$ for all $\mathrm{s} \neq 0 \in \mathrm{K}$.

(3) $\mathrm{A}(\mathrm{p}, \mathrm{q}, \alpha) \leq \mathrm{A}(\mathrm{p}, 0, \alpha)$ and $\mathrm{A}(\mathrm{p}, \mathrm{q}, \alpha) \leq \mathrm{A}(0, \mathrm{q}, \alpha)$ for all $\alpha \in(0, \infty)$.

\section{Lemma 3.10}

The fuzzy norm $N_{A}$ is a non decreasing function.

\section{Proof}

We will show that if $\alpha<\beta$ then $N_{A}(\mathrm{p}, \alpha)<N_{A}(\mathrm{p}, \beta)$. Now suppose that this fails.

That is, $N_{A}(\mathrm{p}, \alpha)>N_{A}(\mathrm{p}, \beta)$ for some $0<\mathrm{t}<\mathrm{s}$. Then, $N_{A}(\mathrm{p}, \alpha) \otimes N_{A}(0, \beta-\alpha) \leq N_{A}(\mathrm{p}, \beta)<N_{A}(\mathrm{p}$, $\alpha$ ), but $N_{A}(0, \beta-\alpha)=1$. Thus, $N_{A}(\mathrm{p}, \alpha)<N_{A}(\mathrm{p}, \alpha)$, which is a contradiction.

\section{Lemma 3.11}

The fuzzy inner product is a non decreasing function.

\section{Proof}

We will show that if $\alpha<\beta$ then $\mathrm{A}(\mathrm{p}, \mathrm{q}, \alpha)<\mathrm{A}(\mathrm{p}, \mathrm{q}, \beta)$. Now, suppose that this fails. That is, $\mathrm{A}(\mathrm{p}, \mathrm{q}$, $\alpha)>\mathrm{A}(\mathrm{p}, \mathrm{q}, \beta)$ for some $0<\alpha<\alpha$. Then,

$\mathrm{A}(\mathrm{p}, \mathrm{q}, \alpha) \otimes \mathrm{A}(0, \mathrm{q}, \beta-\alpha) \leq \mathrm{A}(\mathrm{p}, \mathrm{q}, \beta)<\mathrm{A}(\mathrm{p}, \mathrm{q}, \alpha)$, but $\mathrm{A}(0, \mathrm{q}, \beta-\alpha)=1$.

Thus, $\mathrm{A}(\mathrm{p}, \mathrm{q}, \alpha)<\mathrm{A}(\mathrm{p}, \mathrm{q}, \alpha)$, which is a contradiction.

\section{Theorem 3.12}

Let $(\mathrm{U}, \mathrm{N}, \otimes)$ be a fuzzy normed space. Define

$\mathrm{A}(\mathrm{p}, \mathrm{q}, \alpha+\beta)=\left\{\begin{array}{c}0 \quad \text { if } p=q \text { and } \alpha, \beta \in \mathbb{C} \\ 1 \quad \text { if } p=q \text { and } \alpha, \beta \in(0, \infty) \\ N(p,|\alpha|) \otimes N(q,|\beta|) \text { if } \alpha, \beta \in \mathbb{C}\end{array}\right.$

Then, $(\mathrm{U}, \mathrm{A}, \otimes)$ is a fuzzy inner product space.

\section{Proof}

We will show that all conditions of definition 3.1 are satisfied.

(A1) To show that $\mathrm{A}(\mathrm{p}+\mathrm{q}, \mathrm{w},|\alpha|+|\beta|) \geq \mathrm{A}(\mathrm{p}, \mathrm{w},|\alpha|) \otimes \mathrm{A}(\mathrm{q}, \mathrm{w},|\beta|)$

$\mathrm{A}(\mathrm{p}+\mathrm{q}, \mathrm{w},|\alpha|+|\beta|)=\mathrm{A}(\mathrm{p}+\mathrm{q}, \mathrm{w},|\alpha|+|\beta|+0)=\mathrm{N}(\mathrm{p}+\mathrm{q},|\alpha|+|\beta|) \otimes \mathrm{N}(\mathrm{w}, 0)$

$=\mathrm{N}(\mathrm{p}+\mathrm{q},|\alpha|+|\beta|) \geq \mathrm{N}(\mathrm{p},|\alpha|) \otimes \mathrm{N}(\mathrm{q},|\beta|) \geq[\mathrm{N}(\mathrm{p},|\alpha|) \otimes \mathrm{N}(\mathrm{w}, 0)] \otimes[\mathrm{N}(\mathrm{q},|\beta|) \otimes \mathrm{N}(\mathrm{w}, 0)]=$ $\mathrm{A}(\mathrm{p}, \mathrm{w},|\alpha|) \otimes \mathrm{A}(\mathrm{q}, \mathrm{w},|\beta|)$.

(A2) To show that $\mathrm{A}(\mathrm{p}, \mathrm{q},|\alpha \beta|) \geq \mathrm{A}\left(\mathrm{p}, \mathrm{p},|\alpha|^{2}\right) \otimes \mathrm{A}\left(\mathrm{q}, \mathrm{q},|\beta|^{2}\right)$. Now,

$\mathrm{A}(\mathrm{p}, \mathrm{q},|\alpha \beta|)=\mathrm{N}(\mathrm{p},|\alpha \beta|) \otimes \mathrm{N}(\mathrm{q},|\alpha \beta|)$, but by lemma $2.10,|\alpha \beta|>|\alpha|$, implies

$\mathrm{N}(\mathrm{p},|\beta|)>\mathrm{N}(\mathrm{p},|\alpha|)$, also $\mathrm{N}(\mathrm{q},|\alpha \beta|)>\mathrm{N}(\mathrm{q},|\beta|)$. Thus, $\mathrm{A}(\mathrm{p}, \mathrm{q},|\alpha \beta|)>\mathrm{N}(\mathrm{p},|\alpha|) \otimes \mathrm{N}(\mathrm{q},|\beta|)=\mathrm{A}(\mathrm{p}$, $\left.\mathrm{p},|\alpha|^{2}\right) \otimes \mathrm{A}\left(\mathrm{q}, \mathrm{q},|\beta|^{2}\right)$.

(A3) To show that $\mathrm{A}(\mathrm{p}, \mathrm{q}, \alpha)=\mathrm{A}(\mathrm{q}, \mathrm{p}, \bar{\alpha})$. Let $\mathrm{A}(\mathrm{p}, \mathrm{q}, \alpha)=\mathrm{N}\left(\mathrm{p}, \frac{|\alpha|}{2}\right) \otimes \mathrm{N}\left(\mathrm{q}, \frac{|\alpha|}{2}\right)=\mathrm{N}\left(\mathrm{q}, \frac{|\bar{\alpha}|}{2}\right) \otimes \mathrm{N}(\mathrm{p}$, $\left.\frac{|\bar{\alpha}|}{2}\right)=\mathrm{A}(\mathrm{q}, \mathrm{p}, \bar{\alpha})$.

(A4) To show that $\mathrm{A}(\mathrm{sp}, \mathrm{q}, \mathrm{t})=\mathrm{A}\left(\mathrm{p}, \mathrm{q}, \frac{\alpha}{|s|}\right)$ for all $\mathrm{s} \neq 0 \in \mathrm{K}$. Let $\mathrm{A}(\mathrm{sp}, \mathrm{q}, \alpha)=\mathrm{N}(\mathrm{sp},|\alpha|) \otimes \mathrm{N}(\mathrm{q}, 0)=$ $\mathrm{N}\left(\mathrm{p}, \frac{|\alpha|}{|s|}\right) \otimes \mathrm{N}(\mathrm{q}, 0)=\mathrm{A}\left(\mathrm{p}, \mathrm{q}, \frac{\alpha}{|s|}\right)$.

(A5) $\mathrm{A}(\mathrm{p}, \mathrm{p}, \alpha)=0$ for all $\alpha \in \mathbb{C}-\mathbb{R}$ follows immediately from our definition.

(A6) Now to show that for all $\alpha \in[0, \infty), \mathrm{A}(\mathrm{p}, \mathrm{p}, \alpha)=1$ if and only if $\mathrm{p}=0$. We know that $\mathrm{A}(\mathrm{p}, \mathrm{p}, \alpha)=$ 1 for all $\alpha \geq 1$ if and only if $\mathrm{N}(\mathrm{p}, \sqrt{\alpha})=1$, if and only if $\mathrm{p}=0$.

(A7) it is clear that $\mathrm{A}(\mathrm{p}, \mathrm{q}):, \mathbb{R} \rightarrow \mathrm{I}$ is a continuous function, since $\mathrm{N}(\mathrm{p},$.$) is a continuous function.$

(A8) Finally, to show that $\lim _{t \rightarrow \infty} A(p, q, \alpha)=1$. Let

$\lim _{\alpha \rightarrow \infty} A(u, v, t)=\lim _{\alpha \rightarrow \infty} \mathrm{N}(\mathrm{p},|\alpha|) \otimes \lim _{\alpha \rightarrow \infty} \mathrm{N}(\mathrm{q},|\alpha|)=1 \otimes 1=1$. Hence, $(\mathrm{U}, \mathrm{A}, \otimes)$ is a fuzzy inner product space.

The proof of the next result is similar to the proof of Theorem 3.12 and hence is omitted.

Theorem 3.13

Let $\left(\mathrm{U}, N_{U}, \otimes\right)$ and $\left(\mathrm{V}, N_{V}, \otimes\right)$ be two fuzzy normed spaces. Define

$\mathrm{A}(\mathrm{p}, \mathrm{q}, \alpha+\beta)=\left\{\begin{array}{c}0 \quad \text { if } p=q \text { and } \alpha, \beta \in \mathbb{C} \\ 1 \quad \text { if } p=q \text { and } \alpha, \beta \in(0, \infty) \\ N_{U}(p,|t|) \otimes N_{V}(q,|s|) \text { if } \alpha, \beta \in \mathbb{C}\end{array}\right.$

and $\mathrm{W}=\mathrm{U} \times \mathrm{V}$. Then, $(\mathrm{W}, \mathrm{A}, \otimes)$ is a fuzzy inner product space. 


\section{Definitions 3.14}

Let $(\mathrm{U}, \mathrm{A}, \otimes)$ be a fuzzy inner product space.

(1) The vector $\mathrm{p} \in \mathrm{U}$ is said to be fuzzy orthogonal to the vector $\mathrm{q} \in \mathrm{U}$ and is denoted by $\mathrm{p} \perp^{F} \mathrm{q}$ if $\mathrm{A}(\mathrm{p}$, $\mathrm{q}, \alpha)=1$ for some $\alpha \in(0, \infty)$.

(2) The vector $\mathrm{p} \in \mathrm{U}$ is said to be fuzzy orthogonal to subset $\mathrm{D}$ of $\mathrm{U}$ if $\mathrm{p} \perp^{F} \mathrm{~d}$ for every $\mathrm{d} \neq 0 \in \mathrm{D}$.

(3) $\mathrm{D} \subseteq \mathrm{U}$ is known as a fuzzy orthogonal set if $\mathrm{d} \perp^{F} d_{1}$ for every d, $d_{1} \in \mathrm{D}$.

(4) If $\mathrm{D}$ is a subset of $\mathrm{U}$, then the fuzzy orthogonal complement of $\mathrm{D}$ is known as $D^{\perp^{F}}=\left\{\mathrm{p} \in \mathrm{U}\right.$ : $\mathrm{p} \perp^{F} \mathrm{~d}$ for every $\mathrm{d} \in \mathrm{D}$ \}.

The proof of the next result is straightforward and hence is omitted.

\section{Lemma 3.15}

If $\mathrm{D}$ is a subspace of the fuzzy inner product space $(\mathrm{U}, \mathrm{A}, \otimes)$, then $D^{\perp^{F}}$ is a subspace of $\mathrm{U}$.

\section{Definition 3.16}

The fuzzy inner product space $(\mathrm{U}, \mathrm{A}, \otimes)$ is known as fuzzy complete if every Cauchy sequence $\left(u_{n}\right)$ in $\mathrm{U}$ fuzzy converges to $\mathrm{u} \in \mathrm{U}$.

\section{Theorem 3.17}

If $(\mathrm{U}, \mathrm{A}, \otimes)$ is fuzzy inner product space and $\mathrm{D}$ is a fuzzy complete subspace of $\mathrm{U}$, then for any $\mathrm{u} \in \mathrm{U}$, we can find a unique $\mathrm{d} \in \mathrm{D}$ with $\theta=\sup _{d_{0} \in D} N_{A}\left(\mathrm{u}-d_{0}, \alpha\right)=N_{A}(\mathrm{u}-\mathrm{d}, \mathrm{t})$ for some $\alpha \in(0, \infty)$.

\section{Proof}

First, we show the existence of such $d \in D$. Using the definition of supremum, we can find $\left(d_{n}\right) \in \mathrm{D}$ with $\theta_{n} \rightarrow \theta$ where $\theta_{n}=N_{A}\left(\mathrm{u}-d_{n}, \frac{\alpha}{2}\right)$. We will show that $\left(d_{n}\right)$ is Cauchy. Put $u_{n}=\left(d_{n}-\mathrm{u}\right)$, so we have $N_{A}\left(u_{n}, \frac{\alpha}{2}\right)=\theta_{n}$. Now, $u_{n}-u_{m}=d_{n}-d_{m}$ and when $\mathrm{n}, \mathrm{m}>\mathrm{N} \in \mathbb{N}$, we obtain

$N_{A}\left(d_{n}-d_{m}, \alpha\right)=N_{A}\left(u_{n}-u_{m}, \alpha\right) \geq N_{A}\left(u_{n}, \frac{\alpha}{2}\right) \otimes N_{A}\left(u_{m}, \frac{\alpha}{2}\right) \geq \theta_{n} \otimes \theta_{m}$.

Now, choose $\mathrm{r} \in(0, \infty)$ with $\theta_{n} \otimes \theta_{m}>(1-\mathrm{r})$. This implies that

$N_{A}\left(d_{n}-d_{m}, \alpha\right)>(1-\mathrm{r})$ for each $\mathrm{n}, \mathrm{m}>\mathrm{N}$. Hence, $\left(d_{n}\right)$ is Cauchy in $\mathrm{D}$, but $\mathrm{D}$ is fuzzy complete, so we can find $\mathrm{d} \in \mathrm{D}$ with $d_{n} \rightarrow \mathrm{d}$ and $N_{A}(\mathrm{u}-\mathrm{d}, \mathrm{t}) \geq \theta$. Now

$N_{A}(\mathrm{u}-\mathrm{d}, \alpha)=N_{A}\left(\mathrm{u}-d_{n}+d_{n}-\mathrm{d}, \alpha\right) \geq N_{A}\left(\mathrm{u}-d_{n}, \frac{\alpha}{2}\right) \otimes N_{A}\left(d_{n}-\mathrm{d}, \frac{\alpha}{2}\right) \geq \theta_{n} \otimes 1$. By taking limit to both sides, as $\mathrm{n} \rightarrow \infty,\left(\theta_{n} \otimes 1\right) \rightarrow(\theta \otimes 1)=\theta$, hence,

$N_{A}(\mathrm{u}-\mathrm{d}, \alpha) \geq \theta$, which implies that $N_{A}(\mathrm{u}-\mathrm{d}, \alpha)=\theta$.

Finally we show that such $\mathrm{d}$ is unique. Assume that $\mathrm{d}, d_{0} \in \mathrm{D}$ satisfies both

$N_{A}(\mathrm{u}-\mathrm{d}, \alpha)=\theta$ and $N_{A}\left(\mathrm{u}-d_{0}, \alpha\right)=\theta$. Now,

$N_{A}\left(\mathrm{~d}-d_{0}, \alpha\right)=N_{A}\left(\mathrm{~d}-d_{n}+d_{n}-d_{0}, \alpha\right) \geq N_{A}\left(\mathrm{~d}-d_{n}, \frac{\alpha}{2}\right) \otimes N_{A}\left(d_{n}-d_{0}, \frac{\alpha}{2}\right)$.

By taking limit to both sides as $\mathrm{n} \rightarrow \infty$, we have $N_{A}\left(\mathrm{~d}-d_{0}, \alpha\right)=1$, so $\mathrm{d}=d_{0}$.

\section{Theorem 3.18}

Let $(\mathrm{U}, \mathrm{A}, \otimes)$ be fuzzy inner product space and if, in Theorem 3.17, D is a fuzzy complete subspace of $\mathrm{Y}$, then for fixed $\mathrm{u} \in \mathrm{U}$, the vector $\mathrm{z}=\mathrm{u}-\mathrm{d}$ is fuzzy orthogonal to $\mathrm{Y}$.

\section{Proof}

If $\mathrm{z}$ is not fuzzy orthogonal to $\mathrm{Y}$, then we can find $\mathrm{y} \in \mathrm{Y}$ with $\mathrm{A}(\mathrm{z}, \mathrm{y}, \alpha) \neq 1$. It is clear that $\mathrm{y} \neq 0$, since otherwise $\mathrm{A}(\mathrm{z}, \mathrm{y}, \alpha)=1$. Let $\mathrm{y}$ be chosen so that $\theta \otimes N_{A}(\mathrm{y}, \alpha) \geq \theta$. Now, $N_{A}(\mathrm{z}, \alpha)=N_{A}(\mathrm{u}-\mathrm{d}, \alpha)=\theta$ and $N_{A}(\mathrm{z}-\mathrm{y}, 2 \alpha) \geq N_{A}(\mathrm{z}, \alpha) \otimes N_{A}(\mathrm{y}, \alpha)=\theta \otimes N_{A}(\mathrm{y}, \alpha) \geq \theta$. But this is impossible because we have $\mathrm{z}-\mathrm{y}=\mathrm{u}-\mathrm{d}-\mathrm{y}=\mathrm{u}-y_{1}$ where $y_{1}=(\mathrm{d}+\mathrm{y}) \in \mathrm{Y}$, so that $N_{A}(\mathrm{z}-\mathrm{y}, 2 \alpha) \leq \theta$ by the definition of $\theta$. Hence, $\mathrm{z}$ must be fuzzy orthogonal to $\mathrm{Y}$.

\section{Theorem 3.19}

If $(\mathrm{U}, \mathrm{A}, \otimes)$ is fuzzy complete fuzzy inner product space and $\mathrm{D}$ is a fuzzy closed subspace of $\mathrm{U}$. Then $\mathrm{U}=\mathrm{D} \oplus D^{\perp^{F}}$.

\section{Proof}

By using $\mathrm{U}$ is fuzzy complete and $\mathrm{D}$ is fuzzy closed, and we have that $\mathrm{D}$ is fuzzy complete, then using Theorem 3.18 and Theorem 3.17, for every $\mathrm{u} \in \mathrm{U}$ we can find $\mathrm{d} \in \mathrm{D}$ with $\mathrm{u}=\mathrm{d}+\mathrm{z}$, where $\mathrm{z} \in D^{\perp^{F}}$. To prove that $\mathrm{u}$ has one representation, let $\mathrm{u}=\mathrm{d}+\mathrm{z}$ and $\mathrm{u}=d_{0}+z_{0}$, 
where $\mathrm{d}, d_{0} \in \mathrm{D}$ and $\mathrm{z}, z_{0} \in D^{\perp^{F}}$. Then, $\mathrm{d}-d_{0}=\mathrm{z}-z_{0}$, but $\mathrm{d}-d_{0} \in \mathrm{D}$ whenever $\mathrm{z}-z_{0} \in D^{\perp^{F}}$. This implies that $\mathrm{d}-d_{0} \in\left(\mathrm{D} \cap D^{\perp^{F}}\right)=\{0\}$. Thus, $\mathrm{d}=d_{0}$. Similarly $\mathrm{z}=z_{0}$.

\section{Theorem 3.20}

If $(U, A, \otimes)$ is fuzzy complete fuzzy inner product space and $\mathrm{D}$ is a fuzzy closed subspace of $\mathrm{U}$, then $\mathrm{D}=D^{\perp^{F} \perp^{F}}$.

\section{Proof}

If $\mathrm{d} \in \mathrm{D}$, this implies that $\mathrm{d} \perp^{F} D^{\perp^{F}}$, which implies that $\mathrm{d} \in D^{\perp^{F} \perp^{F}}$. Thus, $\mathrm{D} \subseteq D^{\perp^{F} \perp^{F}}$. On the other hand, assume that $\mathrm{u} \in D^{\perp^{F} \perp^{F}}$ and $\mathrm{u}=\mathrm{d}+\mathrm{z}$, by Theorem 3.19, where $\mathrm{d} \in \mathrm{D} \subseteq D^{\perp^{F} \perp^{F}}$. But, $D^{\perp^{F} \perp^{F}}$ is a subspace of $\mathrm{U}$ and $\mathrm{u} \in D^{\perp^{F} \perp^{F}}$. By our assumption, we also have $\mathrm{z}=\mathrm{u}-\mathrm{d} \in D^{\perp^{F} \perp^{F}}$, so $\mathrm{z}$ is fuzzy orthogonal to $D^{\perp^{F}}$. Now, we have $\mathrm{z} \perp^{F} \mathrm{z}$, thus $\mathrm{z}=0$, thence $\mathrm{u}=\mathrm{d}$, that is $\mathrm{u} \in \mathrm{D}$. Therefore, $D^{\perp^{F} \perp^{F}} \subseteq \mathrm{D}$. Hence, $\mathrm{D}=D^{\perp^{F} \perp^{F}}$.

\section{Conclusions}

In the present paper, we tried to present a definition of fuzzy inner product space, in order to define next fuzzy complete fuzzy inner product spaces, known as fuzzy Hilbert spaces, which will be reasonable enough to be extended. For the extension of fuzzy complete fuzzy inner product spaces, this notion can be very helpful to us and other authors to introduce easier proofs to some results in the ordinary case.

\section{References}

1. Alsina, C. Schweizer, B. Sempi, C. Sklar, A. 1997. On the definition of probabilistic inner product space, Rendiconti di Mathematica, Serie, 17: 115- 127.

2. Su, Y. Wang, X. Gao, J. 2007. Riesz theorem in probabilistic inner product space, Int.Math. Forum. 62: 3073-3078.

3. El-Abyyad, A. M. El-Hamously, H. M.1991. Fuzzy inner product spaces, Fuzzy Sets And Systems 44: 309-326.

4. Kohli, J. K. Kumar, R.1993. On fuzzy inner product spaces and co-inner product spaces, Fuzzy Sets and Systems, 53: 227-232.

5. Kohli, J. K. Kumar, R. 1995. Linear mapping, fuzzy inner product spaces and fuzzy co-inner product spaces, Bull Callcutta Math. Soc. 87(1995): 237-246.

6. Goudarzi, M. Vaezpour, S. M. 2009. On the definition of fuzzy Hilbert spaces and it's applications, J. Nonlinear Sci. Appl. 2: 46-59.

7. Sadeqi, I. Kia, F. 2009. Fuzzy normed linear space and its topological structure, Chaos Solitions and Fractals, 40(5): 2576-2589.

8. Kider, J. R. 2011. On fuzzy normed spaces, Eng. \& Tech. Journal, 29(9): 1790-1795.

9. Kider, J. R. 2011. Completion of fuzzy normed spaces, Eng. \& Tech. Journal, 29(10):2004-2012.

10. Kider, J. R. 2012. New fuzzy normed spaces, J. Baghdad Sci. 9: 559-564.

11. Kider, J. R. Kadhum, N. 2017. Properties of fuzzy norm of fuzzy bounded operators, Iraqi Journal of Science, 58(3A): 1237-1281.

12. Kadhum, N. 2017. On fuzzy norm of a fuzzy bounded operator on fuzzy normed spaces, M.Sc. Thesis, University of Technology, Iraq.

13. Ali, A. 2018. Properties of Complete Fuzzy Normed Algebra, M.Sc. Thesis, University of Technology, Iraq.

14. Kider, J. R. Ali, A. 2018. Properties of fuzzy absolute value on and properties finite dimensional fuzzy normed space. Iraqi Journal of Science, 59(2B): 909-916.

15. Kider, J. R. Gheeab, M. 2019. Properties of a General Fuzzy Normed Space, Iraqi Journal of Science, 60(4): 847-855.

16. Kider, J. R. Gheeab, M. 2019. Properties of The Space GFB(V, U), Journal of AL-Qadisiyah for computer science and mathematics, 11(1): 102-110

17. Kider, J. R. Kadhum, N. 2019. Properties of Fuzzy Compact Linear Operators on Fuzzy Normed Spaces, Baghdad Science Journal, 16(1):104-110.

18. Hussin, E, A. 2019. On some properties of finite dimensional fuzzy anti-normed spaces and Fuzzy anti-inner product spaces, M. Sc. Theses, Department of Mathematics, College of Sciences for Women, University of Baghdad. 